\title{
UNECHTE UND STEIGENDE DIPHTHONGE.
}

Sievers bespricht Phon. ${ }^{3} 143$ das wesen der 'unechten' diphthonge, d. b. jener, deren betonte componente ein laut von geringerer naturlicher schallfulle ist als die unbetonte, und erwähnt auch s. $147 \mathrm{ihr}$ leichtes umschlagen in steigende diphthonge. Durch seine darstellung scheinen mir aber noch nicht alle fragen gelöst, die sich bei diesen bildungen ergeben.

Es handelt sich zunăchst darum, die naturliche schallfulle oder schallstärke der einzelnen laute festzustellen. Dass uberbaupt eine solche abstufung besteht, zeigt schon die erfahrung, dass manche laute auf weitere strecken gehört werden als andere. Woher sie kommt, ist auch klar. Die stärke irgend eines schalles hängt zunächst ab von der schwingungsweite. Bei klängen, die denselben grundton in derselben stärke haben und das sind ja unsere vocale, wenn wir sie mit gleichem atemdruck articulieren - wird daher die verstärkung oder dämpfung gewisser obertöne die schallstärke beeinflussen. Aber noch ein anderes moment wirkt mit, und zwar in noch bedeutenderem masse, scheint mir. Die schallstärke ist auch abhängig von der grösse der schwingenden masse, in unserem falle also, da die masse der stimmbänder dieselbe bleibt, von der im munde zum mittōnen gebrachten luftmenge, die je vach den verschiedenen stellungen sehr verschieden ist.

Es mussten nun einmal wirklich messungen vorgenommen werden; möglich muss dies ja wol sein. Leider aber haben die physiologen, in deren bereich diese aufgabe fällt, den philologen noch nicht diesen dienst geleistet. Auf andrem wege hat Sievers versucht, diese abstufungen festzustellen (Phon. ${ }^{3}$ $181 \mathrm{ff}$ ), und seine methode durfte vorläufig für philologische zwecke ausreichen. Laute mit grosser naturlicher schallfulle eignen sich naturgemäss besser zu silbenträgern als schall- 
schwache laute; zwei vocale werden also leichter zu einem diphthong sich zusammenfügen, d. h. den eindruck des einheitlichen hervorbringen, wenn der schallstärkere silbenträger ist, als wenn das umgekehrte stattfindet. Dies gibt uns ein mittel an die hand die vocale gegenseitig abzuschätzen.

$a$ ist der kräftigste laut. Innerbalb der palatal- und gutturalreihe nimmt die schallstärke mit der grösse der öffnung ab. Was das verhältnis dieser reiben zu einander betrifft, so sind die (gerundeten) gutturalen stärker als die (ungerundeten) palatalen ( $u \underset{\sim}{i}, o_{\sim} e$ fugt sich leichter als $i u, e_{n}$ ), dagegen wol ziemlich gleich stark den gerundeten palatalen, die ubrigens selten in diphthongen vorkommen. Die haupttypen unechter diphthonge sind also ia, ie, io, iu; ea, eo; ua, uo; oa. Dagegen sind $e u, a u, a o$ echte diphthonge. Schwieriger ist die bestimmung bei den guttural-palatalen oder 'mixed vowels' (Sievers $94 \mathrm{ff}$.). Sie machen den eindruck von dumpfen varianten der palatalen oder gutturalen, je nachdem sie ungerundet oder gerundet sind, und werden wol schwächer sein als diese. Der gemischte vocal mit mittlerer zungenhöhe ( $\partial$ in engl. better) scheint mir ungefïhr dieselbe schallstärke zu baben wie der palatale mit hoher zungenstellung, d. h. $i$, und der entsprechende gerundete laut dem $u$ gleichzustehen. Daraus erkläre ich mir, dass $i \partial$ und $\partial i$, uə und $\partial u$ sich gleich leicht aneinanderfugen.

Dass es uberhaupt möglich ist, unechte diphthonge hervorzubringen, hängt damit zusammen, dass auf dem an sich weniger schallkriiftigen laut der grösste atemdruck rubt und dadurch seine stärke erhïht wird (Sievers 191). Aber dieser satz verlangt meines erachtens doch eine nähere bestimmung. Man versuche nur mit unserem gewöhnlichen atemdruck worte wie ahd. liuti, biolan mit (wirklich einsilbigern) iu, io zu sprechen: unser ohr erbält doch immer den eindruck eines steigenden diphthongs. Auch blosse steigerung des atemdrucks hilft nichts; wir mögen diese worte noch so laut sprechen, der akustische effect bleibt derselbe. Der fallende diphthong gelingt erst dann, wenn wir das $i$ sehr stark, alles folgende aber schwach nehmen. Der atemdruck muss also bedeutend herabgesetzt werden, entweder plötzlich beim lubergang vom $i$ zur zweiten componente, eine articulation, diie an sich wol möglich 
ist, aber fur die uns beschäftigenden verhältnisse kaum in betracht kommt, oder aber allmählich, während das $i$ noch tönt, d. b. die erste componente unechter diphthonge muss mit schwach geschnittenem accent gesprochen werden.

Die uns geläufige articulation ist anders. Der diphthong als ganzes trăgt schwach geschnittenen accent, $d . h$. an seinem ende ist der atemdruck bereits viel schwächer als am silbengipfel; aber die erste componente wird in einem augenblick vom gleitlaut zur zweiten componente abgelöst, wo die grösste expirationsstärke kaum voruber ist, geradeso wie sonst kurzer vocal von einem consonanten abgeschnitten wird. Die druckverbältnisse in den silben aut, ait, alt, ast sind vollkommen gleich; uberall wird $\breve{a}$ in oder unmittelbar nach dem augenblick des grōssten druckes abgeschnitten, d. b. mit stark geschnittenem accent gesprochen. Statt $u, i, l, s$ kann aber auch derselbe laut folgen, d. h. a kann so lange gehalten werden als $a u$, ai gedauert hätten; das ergibt die silbe $\bar{a} t$, aber die druckverhältnisse sind keine anderen als frther. Mit einem worte also: wir lassen in allen silben den atemdruck vom silbengipfel aus in derselben weise abnehmen; der schwach geschnittene accent auf lăngen und diphthongen ist nur eine folge ihrer zeitlichen ausdehnung.

Der unterschied des atemdruckes beim silbengipfel und bei dem unmittelbar darauf folgenden laut ist also gering, jedenfalls nicht gross genug um den unterschied in der naturlichen schallstărke z. b. des $i$ und $u$ aufzuwiegen. Das ist die ursache, warum die meisten von uns unwillkiurlich die verschiedenen unechten diphthonge der altgermanischen sprachen als steigende lesen, obwol niemand daran zweifelt, dass sie fallend waren, und dass manche behauptet haben, solche vocalgruppen könnten (fallend) uberhaupt nicht einsilbig gesprochen werden (Sievers 143). Nehmen wir dagegen die erste componente in solchen fällen lang, also $\bar{\imath} u$, so gelingt der fallende diphtliong: am ende des $\bar{\imath}$ ist der atemdruck bereits so schwach, dass das $u$ trotz seiner von natur aus grösseren schallstărke, das $i$ nicht mehr tabertönen kann. Dies leitet uns auf das richtige verfahren um $\imath_{\alpha} u$ hervorzubringen: die erste componente muss, obwol kurz, mit schwach geschnittenem accent hervorgebracht werden. 
Aus diesem satz ergibt sich zunächst, dass unechte diphthonge unmöglich sind in unbetonten silben. Da hier kein accent einwirkt, muss der schallstärkere laut ohne weiteres als silbengipfel empfunden werden. Dasselbe kann auch in betonten silben eintreten, wenn der gravis durch den acut ersetzt wird. Dann tritt die zweite componente so sehr hervor, dass die erste trotz des zuschusses an schallstärke den sie durch den accent erhält, nicht mehr an sie heraureicht; der diphthong wird akustisch ein steigender, während er der articulation nach ein fallender bleibt. Es liegt nun dem sprachgefuhl nahe, dem gehörseindruck zu folgen, den grössten atemdruck auf den stärker klingenden laut zu legen und so den diphthong in einen auch der articulation nach steigenden zu verwandeln. Die sprache wird nicht nur gesprochen, sondern auch gehört, und gewiss ist dieser factor in der sprachentwicklung wirksam gewesen. Aber auch anderes ist möglich: dass der grösste nachdruck auf der ersten componente bleibt und durch andere mittel dem silbenbildungsgesetz, wonach accent und grösste schallstïrke zusammenfallen sollen, geltung verschafft weıden. Einmal kann der diphthong uberhaupt in zwei silben zerfallen. Bei wahrung der einsilbigkeit kann die zweite componente geschwächt werden, entweder indem sie zu einem der unbestimmten und schwachen 'mixed vowels' herabgedrackt wird, oder inden sie in einen weiteren abstand vom silbengipfel ruckt, d. h. die erste componente gedehnt wird. Der so entstandene liberlange diphthong kann wider auf die normale länge gebracht werden durch schwund der zweiten componente.

Die beseitigung der verbindung gravis und kurze kaun endlich auch in der weise gescheben, dass zwar der gravis bestehen bleibt, aber die kïrze verschwipdet durch längung. Das ergebnis ist dasselbe wie in dem \%uletzt erörterten falle.

Unechte diphthonge finden sich nun in den altgermanischen dialekten sehr zahlreich, man kanr geradezu sagen, dass sie bezeichnend für sie sind. Dass sie wirklich fallend waren - mindestens ursprünglich - ist nicht zu bezweifeln. Wir werden daber nach dem oben gesagten annehmen, dass die erste componente den gravis trug. Das scheint mir nun durchaus wahrscheinlich, da ja auch sonst dice kurzen vocale, aller 
wahrscheinlichkeit nach, diesen accent hatten. Darauf weist ausser metrischen erscheinungen die fast uberall eintretende dehnung der kurze in offener silbe; wo sie nicht eintrat, wie in manchen schweizerischen mundarten, ist der schwach geschnittene accent tatsächlich noch vorhanden (Sievers 197). Unmöglich sind solche diphthonge, sagten wir, in unbetonter silbe. Dafür bieten nun das gotische und althochdeutsche, die einzigen dialekte in denen diphthonge in unbetonten silben vorkommen, eine willkommene bestätigung. Während im gotischen $a u$ und $a i$ in allen silben in gleicher weise erscheinen, tritt für das iu der betonten silben in unbetonten ju ein (sunjus, brôprjus). Aehnlich verhält es sich im althochdeutschen (vgl. Braune, Ahd. gramm. $§ 248$ anm. 6). Das -iu in gewissen casus der adjectivdeclination wurde im fränkischen $z u-i n$ und später zu $-u$; im oberdeutschen hielt sich allerdings -iu wie die folgende entwicklung und Notkers schreibung -iu zeigt; aber eben diese schreibung beweist auch, dass -iu nicht in unbetonter silbe stand, sondern einen nebenton trug.

In den betonten silben haben sich diese diphthonge in den einzelnen germanischen sprachen sehr verschieden entwickelt. In den nordischen dialekten trat umschlag zum steigenden diphthong ein, und zwar sehr fruh. Wir müssen also annehmen, dass sehr fruh der gravis durch den acut verdrängt wurde. Dazu stimmt, dass betonte kürze vielfach beseitigt wurde durch dehnung: zunächst im auslaut, später vor gewissen consonantengruppen und endlich in offener silbe (vgl. Noreen in Pauls Grundriss 1, 450 [§ 44]. 470 [§ 105]. 480 [§ 147]). Es scheint mir, dass diesen vorgängen, obwol zeitlich bedeutend entfernt, doch nur eine tendenz zu grunde liegt: die verbindung des schwach geschnittenen accents mit vocalkürze zu beseitigen. Dies geschah entweder durch eintritt des stark geschnittenen accents (in den diphthongen) oder durch längung des vocals. - Ueber ähnliches im fries. vgl. Siebs, Grundr. $1,731 \mathrm{ff}$.

Im englischen haben sich dagegen $e o$, ea lange erbalten, obwol schon sehr früh dehnung der kürze vor gewissen consonantengruppen stattfand: die reihenfolge der einzelnen vorgänge ist also hier umgekehrt. $\dot{e} o$ und $\dot{e} a$ schwinden nicht lange vor der zeit wo die dehnung der kürzen in offener 
silbe eintrat, und zwar durch monophthongierung zu $\bar{e}, \bar{e}$. Im enklitischen séo, héo galt wol schon altengl. eo (vgl. Kluge in Pauls Grundr. 1,840. 849). Im kentischen scheint dagegen früh steigender diphthong eingetreten zu sein, schon im späteren altenglischen finden sich anzeichen dafür (vgl. Sievers, Ags. gr. § 212 anm. 2. Reimann, Die sprache der mittelkentischen evangelien s. $25 \mathrm{ff}$. Danker, Laut- und flexionslehre der mittelkentischen denkmäler s. 7, s. 15. Sweet HES.2 679.683). Die gruppe éo + $w$ ergibt im mittelenglischen sowol eu als ou (ten Brink, Chaucers sprache 39); hier stehen also beide entwicklungen nebeneinander. - Was sich noch später an unechten diphthongen entwickelte, musste zu steigenden werden. Dahin gehört vor allem $i u$ aus me. $\ddot{u}, e u$, $q u$ wie in ne. duke, new, dew (Angl. 14, 287) und ferner jene dialektischen diphthonge, welche Sievers, Phon. ${ }^{3}$ 147 erwähnt. Viele mit $i$ und $u$ beginnende diphthonge aus lebenden mundarten bringt auch Ellis, On Early Engl. Pron. 4, $1309 \mathrm{ff}$. bei, und bemerkt, dass, wenn der accent auf der ersten componente liegt, die zweite gewöhnlich a ist (1310); wenn aber in $i a, u a$ und ähnlichen wirklich deutliches $a$ gesprochen werde, so sei der accent 'very uncertain'; diese diphthonge seien yleichsam im labilen gleichgewichte (in a state of unstable equilibrium) (1311 f.). Das ist offenbar jenes stadium, in welchem der diphthong akustisch steigend, der articulation nach fallend ist.

Auf deutschem boden haben sich die unechten diphthouge sehr lange erhalten. Noch das mittelhochdeutsche hatte uo, ie, aber auch noch gravisbetonung der kurze. Im neuhochdeutschen ist letztere beseitigt, uiud fli: $i e$, $u_{0}$ hat es nach massgabe mitteldeutscher dialekte $\bar{\imath}, \bar{u}$; es ist also wie im englischen monophthongierung eingetreten. Auch ansätze zum ubergang in den steigenden diphthong sind vorhanden in je, jetzt gegenuber nie, itzt; erstere formen sind wol frtih bei enklitischem gebrauch entstanden. Wie es in den mundarten in dieser beziehung aussieht, bleibt $\mathrm{zu}$ untersuchen. Unecbte diphthonge sind $\mathrm{zu}$ erwarten in jenen, welche gravis auf kurze noch bewahrt haben (sie finden sich auch z. b. in der Kerenzer mundart, vgl. Winteler); auch sonst wäre ein läingeres verharren des gravis im diphthong nicht gerade ausgeschlossen, aber recht unwahrscheinlich. Im bairisch-österreichiischen ist ie, uo zu ia, 
uə geworden, der fallende diphthong also durch schwächung der zweiten componente gerettet; in anderen oberdeutschen mundarten finden sich nach Sievers, Phon. 143 zweisilbige vocalgruppen für die alten ie, uo.

$\mathrm{Ob}$ nicht auch ausserhalb des germanischen, namentlich im romanischen, sich ähnliche vorgänge finden, diese frage zu beantworten muss ich berufeneren uberlassen.

WIEN, 10. februar 1891.

KARL LUICK.

\title{
REQUALIVAHANUS.
}

In jahre 1883 wurde bei Blatzheim im reg.-bez. Köln ein römischer votivaltar aus jurakalk aufgepflugt, der sich jetzt im provinzialmuseum zu Bonn befindet. $\mathrm{Er}$ enthält folgende inschrift:

\author{
DEO . REQVALIVAH \\ ANO . Q . APRIANVS \\ FRVCTVS . EX . IMP . PRO \\ SE . ET . SVOS . V.S.L.M.
}

d. i. Deo Requalivàhano $Q($ (uintus) Aprianus Fructus ex imp(erio) pro se et suos $v$ (otum) $s$ (olvit) l(ibens) $m$ (erito).

Die inschrift, von Zangemeister in den Jahrbuchern des vereins von altertumsfreunden im Rheinlande, heft 81 (Bonn, 1886) 8. $78 \mathrm{ff}$. nebst einer photozinkographischen abbildung mitgeteilt und besprochen, ist im ganzen wolerhalten, nur dass der zweite hauptstab des schluss- $H$ in der ersten zeile, die rechte hälfte des letzten $S$ in der zweiten, und endlich der untere teil des kleinen schluss- $o$ in der dritten zeile durch beschädigung des steines zerstört sind. Dass der erste der drei verstlimmelten buchstaben ein $H$ gewesen, unterliegt nach $\mathrm{Z}$. keinem zweifel, 'höchstens könnte noch die zweite (zerstörte) hasta des $\mathrm{H}$ nach oben verlängert gewesen sein $(=h i)$, da die oberfläche auch dort beschädigt ist' (s. 79 f., anm. 2). Nach der form der buchstaben darf man die inschrift Z. zufolge noch ins zweite jb. setzen; die construction der präpos. pro mit dem acc. findet sich auch sonst in inschriften; den gentil- 